\title{
Experience with Active Surveillance of Thyroid Low-Risk Carcinoma in a Developing Country
}

\author{
Alvaro Sanabria ${ }^{1,2}$
}

Background: The incidence of thyroid carcinoma is growing worldwide. More than $50 \%$ of new malignant tumors are classified as classical papillary carcinomas in low-risk category with a low rate of recurrence and high long-term survival. Active surveillance is a strategy to control low-risk tumor evolution with the intention to avoid unnecessary treatments and maintain thyroid function. The aim of this study was to report a cohort of patients with thyroid nodules classified as Bethesda V-VI and who are under active surveillance in Colombia. Methods: A prospective cohort was assembled since January 2015. All patients had been evaluated clinically, biochemically with ultrasonography and fine needle aspiration biopsy previous to the first evaluation. Active surveillance was proposed to patients with asymptomatic low-risk carcinoma $(<1.5 \mathrm{~cm}$, encapsulated, without evidence of lymph node metastasis), independent of sex or age. The strategy included a specific discussion about the surgical option and expected risk of complications, risk of long-term thyroxine support, and the active surveillance strategy. The data on age, sex, reason for an ultrasound examination, ultrasound risk by American Thyroid Association (ATA) classification, size and volume of the nodule, reason to consider active surveillance and follow-up ultrasounds, and surgical decision were recorded prospectively. A Kaplan-Meier curve was constructed for the rate of growth and increase in volume of the nodule, growth $>3 \mathrm{~mm}$ and increase in volume $>50 \%$, and need of operation.

Results: One hundred two patients were analyzed. Seventy-five percent of patients had a nodule smaller than $1 \mathrm{~cm}$. Only 24/102 (23\%) nodules were classified as ATA low risk. Thirty-four of $102(33.3 \%)$ nodules were classified as Bethesda VI and the others were Bethesda V. The median follow-up was 13.9 months, and 32.3\% of patients had more than 24 months of follow-up. Only 11/102 (10.8\%) nodules grew more than $3 \mathrm{~mm}$ and 26/ $102(25.5 \%)$ nodules grew more than $50 \%$ in volume. $12.7 \%$ patients were submitted to surgery. The overall stability rate without growth $>3 \mathrm{~mm}$, without volume increase $>50 \%$, and without surgery at 24 months were $89.8 \%, 77.0 \%$, and $85.5 \%$, respectively

Conclusions: Active surveillance is possible in developing Western countries with similar results to those provided by Asian and American authors.

Keywords: thyroid, thyroid neoplasm, surveillance, thyroidectomy, carcinoma

\section{Introduction}

$\mathbf{T}$ HE INCIDENCE OF thyroid carcinoma is growing worldwide (1). Overdiagnosis is one of the main causes of this accelerated growth. In addition to the incidence increase, there has also been a change in the tumor types. Currently, more than $50 \%$ of new malignant tumors of the thyroid gland have sizes less than $1 \mathrm{~cm}$ and are classified as classical papillary carcinomas, placing them in the low-risk category with a low rate of recurrence and high long-term survival.

Previously, most patients with these low-risk tumors underwent thyroidectomy following the current philosophy of early surgical treatment for most cancers. However, this ap- proach produced a high number of patients with complications, such as laryngeal nerve injury or hypoparathyroidism, who subsequently became dependent on thyroxine support for the remainder of their lives. In the 90s, Japanese surgeons started the practice of active surveillance, and in 2003, publications demonstrated its utility in patients with papillary thyroid low-risk carcinoma without increasing recurrence or compromising long-term survival (2,3). Active surveillance is a strategy to control low-risk tumor evolution by using imaging surveillance with the intention to avoid unnecessary treatments and maintain thyroid function. Nearly concurrent with this, another research group in Japan also demonstrated similar and reproducible results. Only recently, this strategy

\footnotetext{
${ }^{1}$ Department of Surgery, School of Medicine, Universidad de Antioquia, Medellín, Colombia.

${ }^{2}$ Centro de Excelencia en Enfermedades de Cabeza y Cuello (CEXCA), Medellín, Colombia.
} 
has been extended to other Western countries such as the United States (4). Tuttle et al. assembled a cohort of 291 patients and showed that active surveillance can be implemented in the United States (4), replicating the results of Asian authors. To date, other Western groups are introducing this approach in their countries (5). A summary report from Latin America was published in 2018 with 57 patients (6). However, it utilized a short follow-up time and a small sample size.

The aim of this study was to update the results of a cohort of patients with thyroid nodules classified as Bethesda V-VI and who are under active surveillance in Colombia.

\section{Methods}

A prospective cohort from a head and neck cancer center in Medellin, Colombia, was assembled since January 2015. This center covers a population of about 1 million people, is focused on endocrine oncologic surgery, and includes other specialties.

The patients were recruited from the outpatient consult service. They were sent by family physicians, endocrinologists, and general surgeons as potential candidates for thyroidectomy due to an fine needle aspiration biopsy (FNAB) result that was suspicious or malignant (Bethesda classification V and VI). Local institutional review board authorization was provided, and written informed consent was waived due to the descriptive character of the study.

All patients had been evaluated clinically, biochemically (thyrotropin and free thyroxine) with ultrasonography and FNAB previous to the first evaluation by one head and neck surgeon. These evaluations came from different settings, laboratories, and radiologists because the health system in Colombia has a nonintegrated structure. Written ultrasound reports, images, and cytological report from external sources were used to make the decision, and they were not repeated in any case. At the first visit, active surveillance was proposed to every eligible patient with an asymptomatic low-risk carcinoma $(<1.5 \mathrm{~cm}$, encapsulated, without evidence of lymph node metastasis), independent of sex or age. Subsequently, the indication was expanded to older patients ( $>65$ years of age) with severe comorbidities with incidentally discovered nodules even greater than $1.5 \mathrm{~cm}$ (7). There were no patients younger than 18 years.

The strategy included a specific discussion about the surgical option (extent of surgery [most cases, lobectomy]) and expected risk of complications (3\% definitive laryngeal nerve injury and $2 \%$ definitive hypoparathyroidism according to the own rate of complications), risk of long-term thyroxine substitution $(50 \%$ in cases of lobectomy and $100 \%$ in cases of total thyroidectomy), and the active surveillance strategy (evaluation with ultrasound every six months during the first year and once a year thereafter; immediate consultation if clinical symptoms as dysphonia or neck lymph nodes appeared; the possibility of regretting the decision any time after the selection of strategy without any judgment or delay of surgery; the indication of surgery with a nodule growth more than $30 \%$ in the largest dimension or more than $50 \%$ in volume without a new FNAB or suspicious lymph nodes are identified in ultrasound; management of the disease only at the center and advice about avoiding Web exploration about the disease) $(4,8)$. The order of discussion was always the same: first the surgical treatment and then the active surveillance approach up to 2018 when the alternatives order was inverted according to the recommendations of the study by Miyauchi et al. (9). A specific discussion addressed the factors which should be realized to make the decision (e.g., risk-averse or risk-prone personality, anxiety level, fear of surgery, future disease progression, and family support). In all cases, a relative acted as a witness and adviser during the process. Finally, a shared decision was taken after balancing all factors. All tests were not centralized and used the available network of imaging services. As the objective of the study was to assess the efficacy of active surveillance strategy, we did not collect data about patients who rejected the option and only patients who accepted the strategy are reported in this study.

A photographic record of all ultrasound and pathology reports was taken and saved by the principal investigator. The data on age, sex, reason for an ultrasound examination, ultrasound risk by American Thyroid Association (ATA) classification, size and volume of the nodule, reason to consider active surveillance and follow-up ultrasounds, and surgical decision were recorded prospectively in an Excel sheet (Microsoft Corp.). The nodule volume was calculated using the formula (length $\times$ width $\times$ thickness in $\mathrm{mm} \times \pi / 6$ ). In cases where only two measurements were provided, the third was assumed to be the highest. The mean and standard deviation are used to summarize continuous variables, and the percentage and range are used for discrete variables. To compare data, $2 \times 2$ tables were arranged. A Kaplan-Meier curve was constructed for the rate of growth and increase in volume of the nodule, growth $>3 \mathrm{~mm}$ and increase in volume $>50 \%$, and need of operation. Stata software was used for the analysis. A $p$-value $<0.05$ was considered statistically significant.

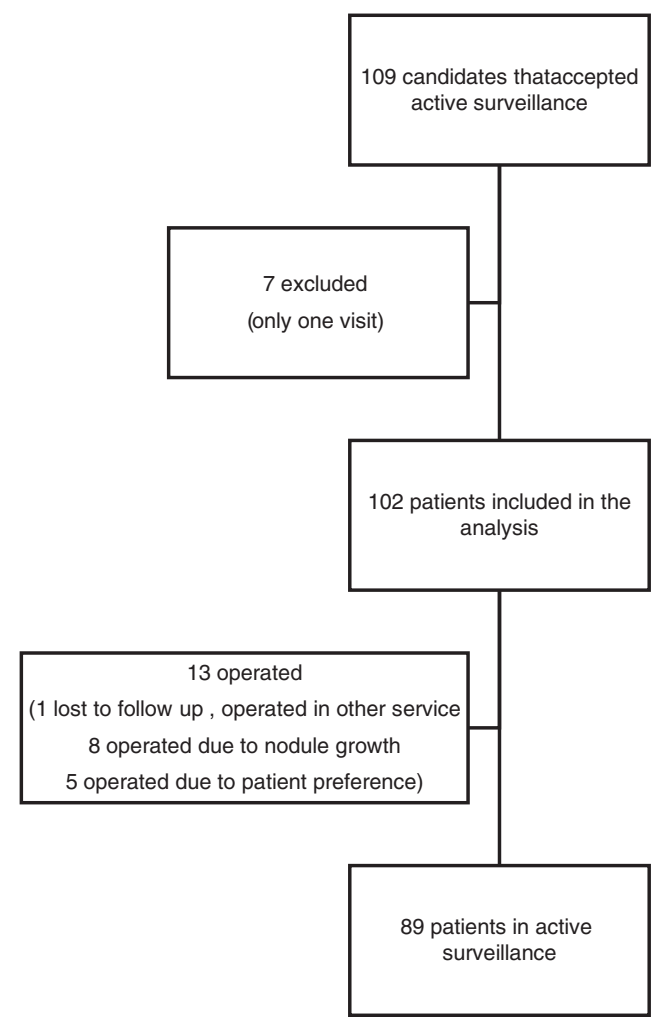

FIG. 1. Flow diagram of included patients. 


\section{Results}

One hundred two patients were analyzed (Fig. 1). The patient characteristics are shown in Table 1. Most patients were women in the fifth decade of life who were diagnosed incidentally with a thyroid nodule. Seventy-five percent of patients had a nodule smaller than $1 \mathrm{~cm}$. Only 24/102 (23\%) nodules were classified as ATA low risk, and 34/102 (33.3\%) nodules were classified as Bethesda VI. The main reason to propose active surveillance was a size $<1 \mathrm{~cm}$ in $64 / 102$ (62.7\%). The median follow-up was 13.9 months $(0.2-112$ months), and $32.3 \%$ of patients had more than 24 months of follow-up.

\section{Nodule behavior}

The nodule size and nodule volume were not significantly different between the initial and final measurements $(p=0.3$ and $p=0.74$, respectively; Fig. 2). Thirty-eight (37.2\%) nodules grew to a mean size of $3.1 \pm 3.5 \mathrm{~mm}$ (median $2 \mathrm{~mm}$ ), but only $11 / 102(10.8 \%)$ nodules grew more than $3 \mathrm{~mm}$ and $15 / 102(14.7 \%)$ nodules grew more than $30 \%$ in the largest diameter. Additionally, 43/102 (42.2\%) nodules increased in volume at a mean of $0.46 \pm 0.86$ (median 0.11 ), but only

Table 1. Demographic and Clinical Characteristics of Patients Who Underwent ACtive Surveillance

\begin{tabular}{|c|c|c|}
\hline Variable & $\mathrm{N}$ & $\%$ \\
\hline Age, years & $50.6 \pm 16.3^{\mathrm{a}}$ & $50(22-86)^{b}$ \\
\hline Age $>60$ years & 29 & 28 \\
\hline Sex (female) & 85 & 83 \\
\hline \multicolumn{3}{|l|}{ Indication of ultrasound } \\
\hline Clinical finding & 7 & 7 \\
\hline $\begin{array}{l}\text { Incidental by } \\
\text { nonthyroid test }\end{array}$ & 1 & 1 \\
\hline $\begin{array}{l}\text { Incidental by a } \\
\text { thyroid ultrasound }\end{array}$ & 94 & 92 \\
\hline $\begin{array}{l}\text { Initial nodule size } \\
\quad \text { (largest diameter) }\end{array}$ & $10.3 \pm 5.8^{\mathrm{a}}$ & $9(3-36)^{b}$ \\
\hline Initial size $>1 \mathrm{~cm}$ & 32 & 25 \\
\hline Initial volume & $0.83 \pm 1.9^{\mathrm{a}}$ & $0.27(0.01-13.3)^{\mathrm{b}}$ \\
\hline Initial volume $>0.5$ & 35 & 33 \\
\hline \multicolumn{3}{|l|}{ ATA ultrasound risk } \\
\hline Low risk & 24 & 23 \\
\hline Medium risk & 56 & 55 \\
\hline High risk & 22 & 22 \\
\hline \multicolumn{3}{|l|}{ Bethesda classification } \\
\hline V & 68 & 67 \\
\hline VI & 34 & 33 \\
\hline \multicolumn{3}{|c|}{ Reason for active surveillance } \\
\hline $\begin{array}{l}\text { Old patient/severe } \\
\text { comorbidity }\end{array}$ & 9 & 9 \\
\hline Nodule $<1 \mathrm{~cm}$ & 64 & 63 \\
\hline $\begin{array}{l}\text { Desire of } \\
\text { surveillance }\end{array}$ & 29 & 28 \\
\hline $\begin{array}{l}\text { Actual number of visits } \\
\text { Time of follow-up } \\
\text { (months) }\end{array}$ & $\begin{array}{c}2.4 \pm 1.4^{\mathrm{a}} \\
20.8 \pm 19.8^{\mathrm{a}}\end{array}$ & $\begin{array}{c}2(1-6)^{\mathrm{b}} \\
13.9(0.2-112)^{\mathrm{b}}\end{array}$ \\
\hline
\end{tabular}

\footnotetext{
${ }^{\mathrm{a}} \mathrm{Mean} \pm \mathrm{SD}$.

${ }^{\mathrm{b}}$ Median (range).

ATA, American Thyroid Association; SD, standard deviation.
}

$26 / 102(25.5 \%)$ nodules grew more than $50 \%$ in volume. Nine of $70(12.9 \%)$ patients with nodules $<1 \mathrm{~cm}$ showed an increase to more than $1 \mathrm{~cm}$, but $11 / 33(34.4 \%)$ patients with nodules $>1 \mathrm{~cm}$ showed a decrease to less than $1 \mathrm{~cm}$. Nodules $>1 \mathrm{~cm}$ had lower increases in size and volume than nodules $\leq 1 \mathrm{~cm}$.

Thirteen (12.7\%) patients were submitted to surgery ( 8 due to nodule growth and 5 for other reasons). All had a papillary carcinoma, 5 were treated with lobectomy and 8 with total thyroidectomy. One patient needed a therapeutic central neck dissection, other a prophylactic neck dissection, and a third, a lateral neck dissection due to intraoperative findings of lymph node metastasis. Eight patients were operated during the first year of follow-up (seven because nodule growth). The overall stability rate without growth $>3 \mathrm{~mm}$, without volume increase $>50 \%$, and without surgery at 24 months were $89.8 \%, 77 \%$, and $85.5 \%$, respectively (Fig. 3A-C). When stratified by age $<40,41-60$, and $>60$ years, the frequency of growth $>3 \mathrm{~mm}$ was $17.5 \%, 9.0 \%$, and $4.0 \%$, respectively.

\section{Agreement between growth $>3 \mathrm{~mm}$ and increase in volume $>50 \%$}

Table 2 shows the agreement between patients with nodules with growth $>3 \mathrm{~mm}$ and those who showed an increase in volume $>50 \%$. The volume increase was overestimated by $16.5 \%$ compared with the largest diameter growth.

\section{Discussion}

The main cause of the increase in new cases of thyroid cancer is overdiagnosis (10). This phenomenon is mainly due to the overuse of thyroid ultrasonography in most asymptomatic patients. Data from cancer registries have shown that most new tumors occur in nodules smaller than $1.5 \mathrm{~cm}$ that are usually not easily detected by physical examination (1). The consequence is that many patients, especially younger females with small nodules, became cancer patients. Additionally, a new population of older patients with multiple comorbidities with an incidentally discovered thyroid nodule is growing.

These patients with low-risk cancers are candidates to thyroidectomy. ATA guidelines recommend that patients with intrathyroid nodules smaller than $1-4 \mathrm{~cm}$ with a V-VI Bethesda classification undergo at least lobectomy (11). Thus, many patients with incidentally discovered nodules will be at risk of surgical complications and will become dependent on levothyroxine substitution. This situation can even worsen in older patients with multiple comorbidities where the anesthetic and postoperative complications are more frequent (12). However, many studies have demonstrated that the effect of these small tumors on overall survival is minimal (1). This discrepancy offered an opportunity to explore new alternatives such as active surveillance.

Active surveillance was evaluated initially by Ito et al. from Kuma Hospital in 2003 (2). In an initial cohort of 162 patients with low-risk thyroid tumors smaller than $1 \mathrm{~cm}$, they found a growth rate of $10 \%$ and a frequency of lateral neck lymph node metastasis of $1.2 \%$ after 46 months of follow-up. Further reports with many patients and a longer follow-up confirmed the initial data of stability in most low-risk carcinomas (13-17). Simultaneously, Sugitani and colleagues assembled a similar cohort of 244 patients and showed an 
FIG. 2. Initial and final measurements of the largest diameter and volume of patient nodules who underwent active surveillance.
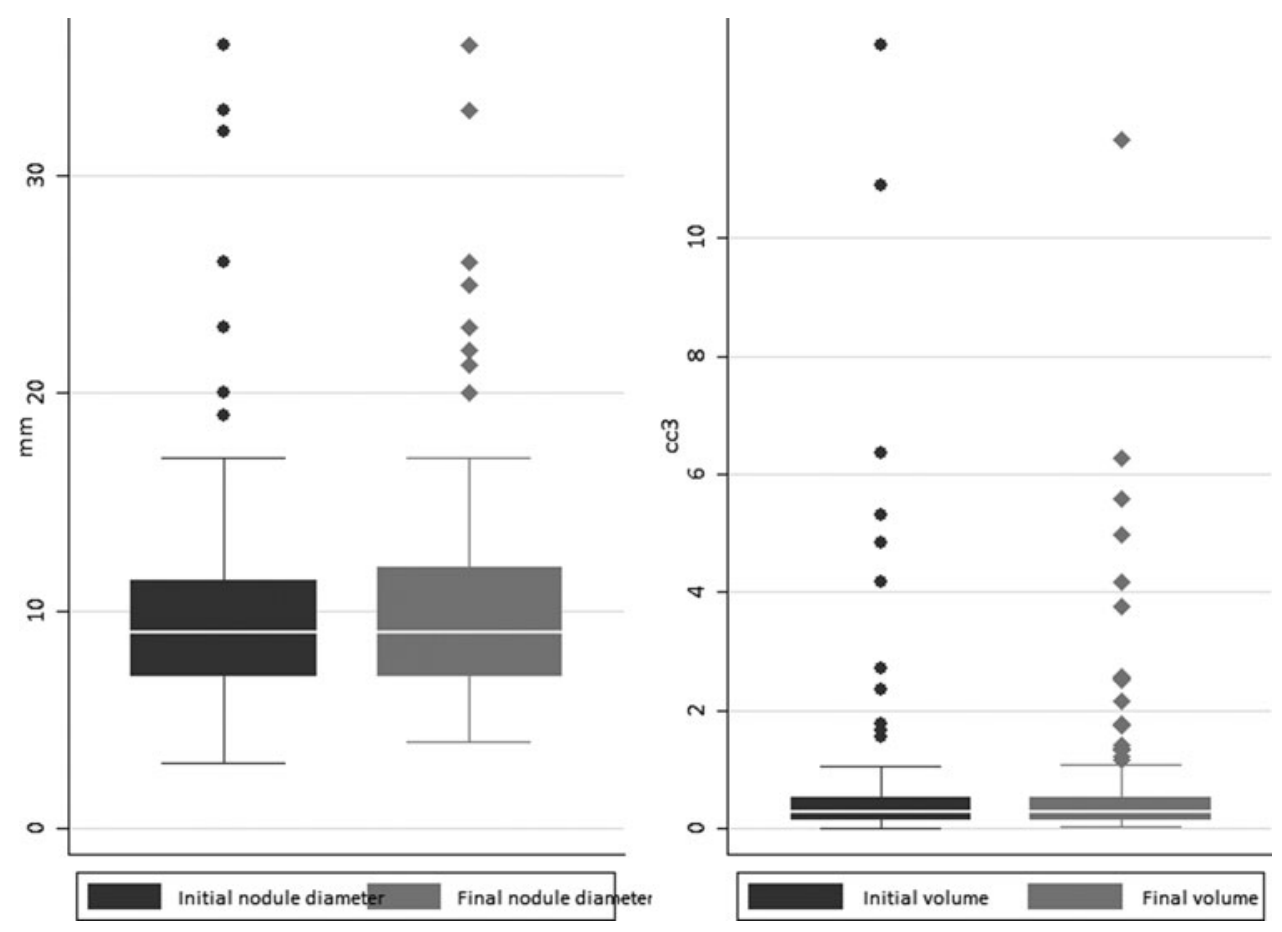

A

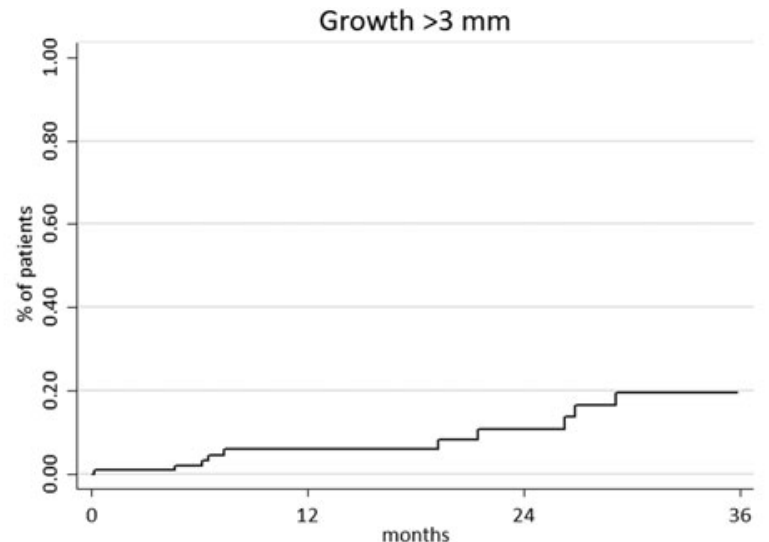

Number at risk
57

34
B

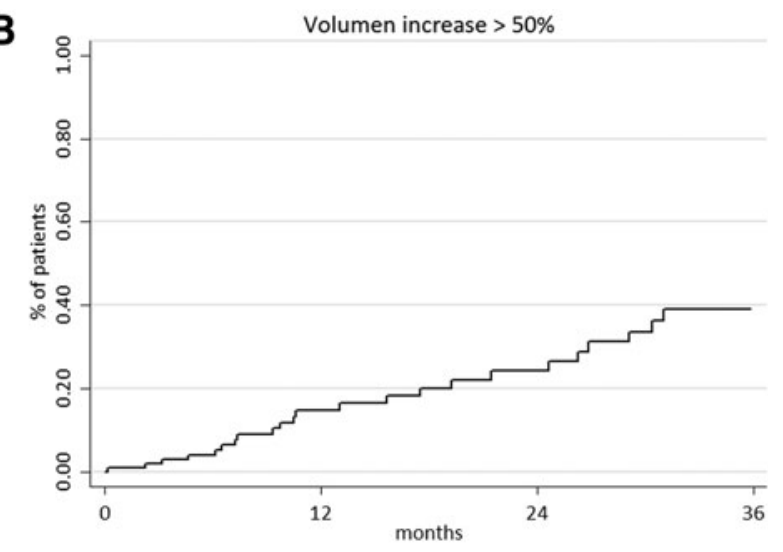

Number at risk
57

34

17

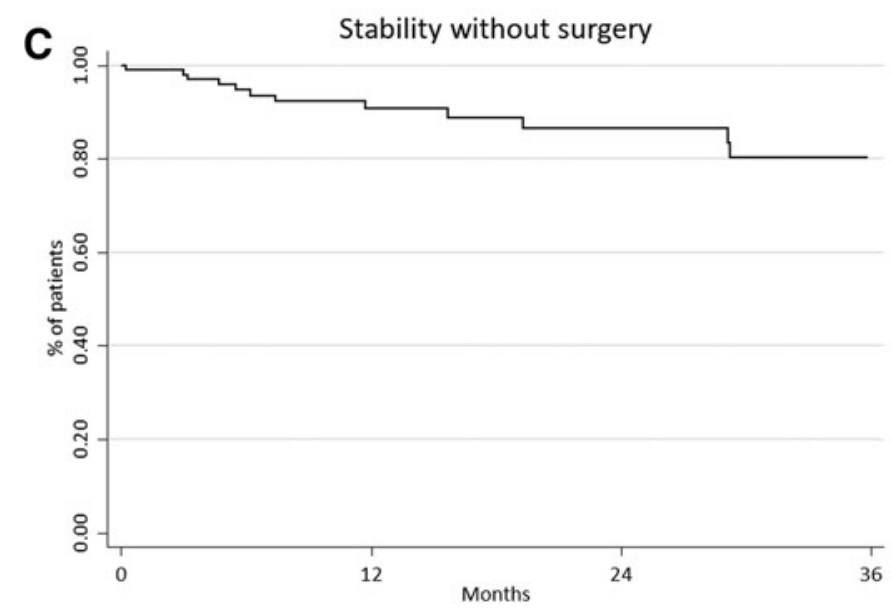

FIG. 3. Behavior of nodules that underwent active surveillance. (A) Nodules that grew $>3$ mm. (B) Nodules with an increased volume $>50 \%$. (C) Nodules that needed surgery. 
Table 2. Comparison Between the Size Growth $>3$ MM AND VOLUME INCREASE $>50 \%$

\begin{tabular}{lccr}
\hline Increase & Volume $\leq 50 \%$ & Volume $>50 \%$ & \\
\hline Growth $\leq 3 \mathrm{~mm}$ & 76 & 15 & 91 \\
Growth $>3 \mathrm{~mm}$ & 0 & 11 & 11 \\
Total & 76 & 26 & 102 \\
\hline
\end{tabular}

increase higher than $3 \mathrm{~mm}$ in $7 \%$ and a frequency of lymph node metastasis of $0.9 \%(7,18)$. Other experiences have been published from Korea $(19,20)$. However, despite the important number of patients and long follow-up, the strategy of active surveillance was restricted to Asia and was not easily adopted in Western countries. Some authors argued that surveillance was not possible in other countries due to the risk of occult extrathyroidal extension or microscopic central lymph node metastasis, the differences in patients' genetics, and perspectives and difficulties of long-term follow-up compared with surgery $(21,22)$. In 2017, Tuttle et al. reported the first experience with active surveillance in the United States with 291 patients (4). They found that only $3.8 \%$ of patients had a growth $>3 \mathrm{~mm}$ and an operation rate of $3.5 \%$. Short reports were also published in Germany, Argentina, and Colombia $(6,23,24)$. However, this last report included few patients with a short follow-up. Other groups from $\mathrm{Ca}$ nada and Korea have explored the use of active surveillance; however, until now, no published results are available $(5,25)$. Therefore, this is the first complete report of active surveillance in Latin America with long-term follow-up.

This study recruited 102 patients with similar characteristics to other reports. Besides the common patient type, a new group of patients is emerging. Almost one-third of our patients were older than 60 years, where incidental findings were a product of imaging tests as computed tomography (CT), magnetic resonance imaging (MRI) and positron emission tomography-computed tomography (PET-CT), and 10 of these patients had severe comorbidities, sufficient to consider any surgical treatment life-threatening. This subgroup includes patients with the lowest probability of tumor growth and with a low likelihood of progression when included in an active surveillance strategy, and they correspond to the ideal group of candidates according to Brito et al. $(17,26)$.

The behavior of the nodules was similar to other reports. Although $37 \%$ of nodules grew in the largest diameter, only $11 \%$ of them grew more than $3 \mathrm{~mm}$. After two years, Ito et al. (2) found that only $21 \%$ of patients had an increase larger than $2 \mathrm{~mm}$, and Sugitani et al. (27) and Tuttle et al. (4) reported that only $7 \%$ and $3.8 \%$ grew more than $3 \mathrm{~mm}$, respectively. Regarding the volume, the observed rate of increase was higher in our study, with $42 \%$ showing any increase and $25 \%$ demonstrating an increase $>50 \%$. Tuttle et al. (4) found a rate of increase in volume $>50 \%$ in $11 \%$ of patients at 2 years, while Oh et al. (20) found a rate of $23 \%$. The choice between size $(27,28)$ or volume $(4,20)$ increases as the main criterion to make a surgical decision is variable in studies. In this study, the volume overestimates the growth and could induce surgical treatment in almost $16 \%$ of patients who would not need it if size increase had been used, so is clear that volume would be too sensitive. Moreover, the change of volume is consequently greater for originally small tumors than for originally large tumors.
Because some studies suggest that the upper limit to indicate surgery is $1 \mathrm{~cm} \mathrm{(7),} \mathrm{we} \mathrm{explored} \mathrm{the} \mathrm{change} \mathrm{in} \mathrm{the} \mathrm{size}$ of nodules based on the initial diameter. Although $13 \%$ of nodules smaller than $1 \mathrm{~cm}$ grew to more than $1 \mathrm{~cm}$, one-third of nodules that started with a diameter higher than $1 \mathrm{~cm}$ returned to a size below this threshold. Ito et al. demonstrated that tumors larger than $7 \mathrm{~mm}$ tended to enlarge more frequently than those smaller than $7 \mathrm{~mm}$ (28). However, larger nodules tend to grow slower than small nodules. This situation suggests that a unique measurement that shows a growth in the nodule size should not be an absolute indication for surgery and that observation with a shorter interval for ultrasound evaluations could avoid unnecessary surgery in almost $33 \%$ of patients.

The findings obtained in this cohort also confirm previous results of the stability and security of active surveillance. At 2 years of follow-up, $89 \%$ of nodules remained stable if the size is used as the criterion, or $77 \%$ if the volume is the criterion. These results are similar to those previously reported $(4,17,18,28)$. However, for younger patients, the rate of growth was higher (19\% vs. $9 \%)$. Eight of the 13 patients were operated during the first year of follow-up because of nodule growth, but this situation is not widely explored in published trials. This may be the result of selection criteria that only considered to include patients with a minimal follow-up. Early failures must also be considered failures of the active surveillance strategy. These two situations can be a result of selection bias, with a probable inclusion of inappropriate candidates to active surveillance.

Five of $13(38 \%)$ patients underwent surgery for reasons not related with nodule enlargement. Other series have shown a similar $(20,29)$ or a higher rate $(13,28)$ of operations. The proposed arguments to explain the reasons for surgical intervention include failure to identify appropriate candidates, physician/patient anxiety, and the difficulties to implement this new alternative in unintegrated health systems (30). Brito et al. proposed a framework to select the best candidates for active surveillance and endocrinologists and surgeons involved in thyroid cancer management should be familiar with these recommendations (26). Nickel et al. have found that terminology used to explain the ultrasonographic and pathological findings to patients have an important effect on the selection of treatment $(31,32)$. The use of the word cancer is a driver to propose or request surgery, and it should be replaced by other clinical terms that better reflect the biological behavior of low-risk thyroid carcinoma. However, even after an informed decision had been taken by the patient, some studies have shown that pressure and uninformed opinions of physicians, relatives, and friends that ignore the results and advantages of active surveillance increase the anxiety of patients and results in regretting the choice of active surveillance (33-35). The relative success of this study is based on a long and dedicated discussion with the patient about the pros and cons of the alternative, a relationship of trust where the patient does not feel guilty or anxious if he/she changes his/her mind about surveillance, and the close participation of family members in the decision.

Finally, health system barriers (26), such as the lack of knowledge about indications and results of active surveillance by health personnel, lack of integrality in the diagnosis and management of patients with low-risk thyroid carcinoma, intolerance to uncertainty by some members of the medical 
team, fear of lawsuits, and the position of scientific societies against the strategy, which are most common in developing countries, must be faced with an educational strategy to make this option visible, show the results, discuss its advantages and limitations, and transcend concerns. Skepticism is common and healthy when new paradigms are born, and medical history has taught that facts and patience are the key to the change (36).

This study has weaknesses that must be recognized. The follow-up is short when compared with other series, and the growth rate of lesions can change with time. The ultrasound evaluation was not centralized and made by nondedicated thyroid radiologists, which can decrease the reliability of the results. However, this study is the first that applies the strategy in a developing country and outside major academic reference centers, and these results could reflect the behavior of active surveillance in a real-world setting.

In conclusion, active surveillance is possible in developing Western countries with similar results to those provided by Asian and American authors if shared decision-making and follow-up are implemented.

\section{Author Disclosure Statement}

No competing financial interests exist.

\section{Funding Information}

No funding was received.

\section{References}

1. Davies L, Welch HG 2014 Current thyroid cancer trends in the United States. JAMA Otolaryngol Head Neck Surg 140: 317-322.

2. Ito Y, Uruno T, Nakano K, Takamura Y, Miya A, Kobayashi K, Yokozawa T, Matsuzuka F, Kuma S, Kuma K, Miyauchi A 2003 An observation trial without surgical treatment in patients with papillary microcarcinoma of the thyroid. Thyroid 13:381-387.

3. Sugitani I, Fujimoto Y 1999 Symptomatic versus asymptomatic papillary thyroid microcarcinoma: a retrospective analysis of surgical outcome and prognostic factors. Endocr J 46:209-216.

4. Tuttle RM, Fagin JA, Minkowitz G, Wong RJ, Roman B, Patel S, Untch B, Ganly I, Shaha AR, Shah JP, Pace M, Li D, Bach A, Lin O, Whiting A, Ghossein R, Landa I, Sabra M, Boucai L, Fish S, Morris LGT 2017 Natural history and tumor volume kinetics of papillary thyroid cancers during active surveillance. JAMA Otolaryngol Head Neck Surg 143:1015-1020.

5. Sawka AM, Ghai S, Tomlinson G, Rotstein L, Gilbert R, Gullane P, Pasternak J, Brown D, de Almeida J, Irish J, Chepeha D, Higgins K, Monteiro E, Jones JM, Gafni A, Goldstein DP 2018 A protocol for a Canadian prospective observational study of decision-making on active surveillance or surgery for low-risk papillary thyroid cancer. BMJ Open 8:e020298.

6. Sanabria A 2018 Active surveillance in thyroid microcarcinoma in a Latin-American Cohort. JAMA Otolaryngol Head Neck Surg 144:947-948.

7. Sakai T, Sugitani I, Ebina A, Fukuoka O, Toda K, Mitani H, Yamada K 2019 Active surveillance for T1bN0M0 papillary thyroid carcinoma. Thyroid 29:59-63.
8. Ito Y, Miyauchi A, Oda H 2018 Low-risk papillary microcarcinoma of the thyroid: a review of active surveillance trials. Eur J Surg Oncol 44:307-315.

9. Miyauchi A, Ito Y, Oda H 2018 Insights into the management of papillary microcarcinoma of the thyroid. Thyroid 28:23-31.

10. Sanabria A, Kowalski LP, Shah JP, Nixon IJ, Angelos P, Williams MD, Rinaldo A, Ferlito A 2018 Growing incidence of thyroid carcinoma in recent years: factors underlying overdiagnosis. Head Neck 40:855-866.

11. Haugen BR, Alexander EK, Bible KC, Doherty GM, Mandel SJ, Nikiforov YE, Pacini F, Randolph GW, Sawka AM, Schlumberger M, Schuff KG, Sherman SI, Sosa JA, Steward DL, Tuttle RM, Wartofsky L 20162015 American Thyroid Association Management Guidelines for adult patients with thyroid nodules and differentiated thyroid cancer: the American Thyroid Association Guidelines Task Force on thyroid nodules and differentiated thyroid cancer. Thyroid 26:1-133.

12. McIntire JB, McCammon S, Mong ER 2018 Endocrine surgery in the geriatric population. Otolaryngol Clin North Am 51:753-758.

13. Ito $\mathrm{Y}$, Miyauchi A, Inoue H, Fukushima M, Kihara M, Higashiyama T, Tomoda C, Takamura Y, Kobayashi K, Miya A 2010 An observational trial for papillary thyroid microcarcinoma in Japanese patients. World J Surg 34:28-35.

14. Ito Y, Miyauchi A, Kobayashi K, Miya A 2014 Prognosis and growth activity depend on patient age in clinical and subclinical papillary thyroid carcinoma. Endocr J 61:205-213.

15. Ito Y, Miyauchi A, Kihara M, Higashiyama T, Kobayashi K, Miya A 2014 Patient age is significantly related to the progression of papillary microcarcinoma of the thyroid under observation. Thyroid 24:27-34.

16. Ito Y, Miyauchi A, Oda H, Kobayashi K, Kihara M, Miya A 2016 Revisiting low-risk thyroid papillary microcarcinomas resected without observation: was immediate surgery necessary? World J Surg 40:523-528.

17. Miyauchi A, Kudo T, Ito Y, Oda H, Sasai H, Higashiyama T, Fukushima M, Masuoka H, Kihara M, Miya A 2018 Estimation of the lifetime probability of disease progression of papillary microcarcinoma of the thyroid during active surveillance. Surgery 163:48-52.

18. Sugitani I, Fujimoto Y 2010 Management of low-risk papillary thyroid carcinoma: unique conventional policy in Japan and our efforts to improve the level of evidence. Surg Today 40:199-215.

19. Kwon H, Oh HS, Kim M, Park S, Jeon MJ, Kim WG, Kim WB, Shong YK, Song DE, Baek JH, Chung KW, Kim TY 2017 Active surveillance for patients with papillary thyroid microcarcinoma: a single center's experience in Korea. J Clin Endocrinol Metab 102:1917-1925.

20. Oh HS, Kwon H, Song E, Jeon MJ, Kim TY, Lee JH, Kim WB, Shong YK, Chung KW, Baek JH, Kim WG 2019 Tumor volume doubling time in active surveillance of papillary thyroid carcinoma. Thyroid 29:642-649.

21. Sturgis EM, Sherman SI 2010 Should papillary thyroid carcinoma be observed?: a word of caution. Arch Otolaryngol Head Neck Surg 136:444-446.

22. Paparodis RD, Bantouna D, Imam S, Jaume JC 2019 The non-interventional approach to papillary thyroid microcarcinomas. An "active surveillance" dilemma. Surg Oncol 29:113-117.

23. Dralle H, Weber F 2019 Active surveillance versus primary surgery for papillary microcarcinoma of the thyroid gland [in German]. Chirurg 90:110. 
24. Smulever A, Bueno F, Abelleira E, Pitoia F 2018 Active surveillance: A new alternative in patients with well-differentiated thyroid carcinoma [in Spanish]. In: Endocrinología FAdSd (ed) XII Congreso FASEN, Mar del Plata, Argentina.

25. Moon JH, Kim JH 2018 Study Protocol of Multicenter Prospective Cohort Study of Active Surveillance on Papillary Thyroid Microcarcinoma (MAeSTro). Endocrinol Metab (Seoul) 33:278-286.

26. Brito JP, Ito Y, Miyauchi A, Tuttle RM 2016 A clinical framework to facilitate risk stratification when considering an active surveillance alternative to immediate biopsy and surgery in papillary microcarcinoma. Thyroid 26:144-149.

27. Sugitani I, Toda K, Yamada K, Yamamoto N, Ikenaga M, Fujimoto Y 2010 Three distinctly different kinds of papillary thyroid microcarcinoma should be recognized: our treatment strategies and outcomes. World J Surg 34:12221231.

28. Ito $\mathrm{Y}$, Tomoda $\mathrm{C}$, Uruno $\mathrm{T}$, Takamura $\mathrm{Y}$, Miya A, Kobayashi K, Matsuzuka F, Kuma K, Miyauchi A 2004 Papillary microcarcinoma of the thyroid: how should it be treated? World J Surg 28:1115-1121.

29. Sugitani I, Fujimoto Y, Yamada K 2014 Association between serum thyrotropin concentration and growth of asymptomatic papillary thyroid microcarcinoma. World J Surg 38:673-678.

30. Haymart MR, Miller DC, Hawley ST 2017 active surveillance for low-risk cancers-a viable solution to overtreatment? N Engl J Med 377:203-206.

31. Nickel B, Brito JP, Barratt A, Jordan S, Moynihan R, McCaffery K 2017 Clinicians' views on management and terminology for papillary thyroid microcarcinoma: a qualitative study. Thyroid 27:661-671.

32. Nickel B, Brito JP, Moynihan R, Barratt A, Jordan S, McCaffery K 2018 Patients' experiences of diagnosis and management of papillary thyroid microcarcinoma: a qualitative study. BMC Cancer 18:242.

33. Davies L, Hendrickson CD, Hanson GS 2017 Experience of US patients who self-identify as having an overdiagnosed thyroid cancer: a qualitative analysis. JAMA Otolaryngol Head Neck Surg 143:663-669.

34. Davies L, Roman BR, Fukushima M, Ito Y, Miyauchi A 2019 Patient experience of thyroid cancer active surveillance in Japan. JAMA Otolaryngol Head Neck Surg 145:363-370.

35. Rittenmeyer L, Huffman D, Alagna M, Moore E 2016 The experience of adults who choose watchful waiting or active surveillance as an approach to medical treatment: a qualitative systematic review. JBI Database Syst Rev Implement Rep 14:174-255.

36. Aronson J 2015 Five types of skepticism. BMJ 350:h1986.

Address correspondence to:

Alvaro Sanabria, $P h D$

Department of Surgery

School of Medicine

Universidad de Antioquia

Cra. 51d \#62-29

Medellín 050010

Colombia

E-mail: alvarosanabria@gmail.com 